\title{
Epidemiology of Yeast Colonization in the Intensive Care Unit
}

\author{
S.A. Hedderwick, M.J. Lyons, M. Liu, J.A. Vazquez, C.A. Kauffman
}

\begin{abstract}
In order to investigate the epidemiology of colonization and possible transmission of yeasts among patients and healthcare workers in adult intensive care units (ICUs), 194 patients were followed for a mean of $9 \pm 11$ days and 63 healthcare workers were followed for a mean of $132 \pm 52$ days. Among the patients, 142 (73\%) were colonized by yeast, with Candida albicans being the species most commonly recovered. Most patients $(65 \%)$ were already colonized with yeast upon admission to the intensive care unit; only $17 \%$ became colonized after admission. Persistent colonization occurred in $51(55 \%)$ of 92 patients who had more than three cultures performed; in $75 \%$ of them, colonization persisted with the same strain of Candida albicans or Candida glabrata. Bacterial infection in the month preceding entry into the ICU was the only risk factor significantly associated with yeast colonization. Among the healthcare workers, yeasts were isolated from $42(67 \%)$. Candida albicans was most frequently recovered from the oropharynx (19\% of occasions), and Candida parapsilosis was most frequently found on hands ( $8 \%$ of occasions). Persistent colonization of the oropharynx occurred in only six healthcare workers, and none had persistence of yeasts on hands. In this non-outbreak setting, 5 (4\%) of 123 patient/healthcare worker interactions that were linked epidemiologically yielded the same strain of Candida albicans, providing evidence for possible cross-transmission. No similar link was found between healthcare worker-patient interactions and colonization with Candida glabrata or Candida parapsilosis.
\end{abstract}

\section{Introduction}

The incidence of serious Candida infections in critically ill patients in intensive care units (ICU) is increasing; currently, Candida species constitute the fourth most common nosocomial bloodstream isolate in the USA

S.A. Hedderwick, M.J. Lyons, M. Liu, C.A. Kauffman (凶) Division of Infectious Diseases, Ann Arbor Veterans Affairs Healthcare System, University of Michigan Medical School, 2215 Fuller Road, Ann Arbor, MI 48105, USA e-mail: ckauff@umich.edu

J.A. Vazquez

Division of Infectious Diseases, Wayne State University School of Medicine, Detroit, MI, USA

Present address: S.A. Hedderwick, Royal Victoria Hospital, Belfast, United Kingdom

Present address: M.J. Lyons, Grand Valley State University, Allendale, MI, USA
$[1,2]$. The epidemiology of Candida infections is still controversial. It is generally believed that infection arises from invasion by the patient's own endogenous colonizing flora and that those strains of Candida that cause infection are the same as those previously found colonizing patients [3-5]. However, several reports of outbreaks of Candida infections support exogenous acquisition of the infecting yeast strains in some cases [6-13].

Several studies have documented that $60-70 \%$ of patients in ICUs are colonized with Candida spp. [14, 15]. Fewer studies, however, have assessed the prevalence of yeast colonization among healthcare workers in that same setting. Although one study found that $58 \%$ of ICU nurses carried yeasts of any type on their hands [16], others have noted low carriage rates for yeasts $[13,15]$.

The investigation of possible transmission of yeasts between patients and healthcare workers has been 
hampered by the lack of a reliable typing system to evaluate strain relatedness. Previous typing systems that relied on phenotypic or serologic differences were not sensitive enough. Recently, molecular typing systems for yeasts, including contour-clamped homogenous electric field (CHEF) electrophoresis and random amplified polymorphic DNA (RAPD) techniques, have facilitated the identification of strain types carried by individuals and have helped clarify the modes of transmission [17]. Using these techniques, we prospectively studied the patterns of yeast colonization and sought to determine whether yeast strains were being transmitted between healthcare workers and patients in the ICU setting.

\section{Materials and Methods}

Facility. The Ann Arbor Veterans Affairs Medical Center is a 200-bed acute-care veterans hospital with a long-term-care unit attached to the hospital. There are three ICU's: one seven-bed medical ICU with single rooms only, one seven-bed thoracic ICU with single rooms only, and one eight-bed surgical ICU with two single rooms and a six-bed open unit.

Study Participants. From 1 May 1995 through 31 October 1995, all patients who were expected to remain in one of the ICUs for at least 3 days were eligible for the study. If patients were readmitted to the ICU on a second occasion, they were not reenrolled into the study. During the same study period, healthcare workers caring for the patients in the ICUs were asked to participate; approximately 40 of them worked in the medical ICU only, and another 60 rotated through the thoracic ICU and the surgical ICU. All nurses worked 12-h shifts. Generally, each healthcare worker was assigned one or two patients according to the complexity of the patient's needs; occasionally, more than one healthcare worker cared for a single patient.

Study Design. Demographic and microbiologic data were obtained for each patient every 3 days while they were in the ICU and once more approximately 3 days following their discharge from the ICU. At entry and with each sampling, the following data were obtained: age, sex, underlying diseases, prior surgical procedures, parenteral nutrition, immunosuppressive therapy, recent history of bacterial or fungal infection, and antimicrobial treatment. Rectal and buccal mucosa were sampled for the presence of yeasts every 3 days. For healthcare workers, buccal mucosa and hands were sampled for the presence of yeasts every 3 days as their shift schedules permitted. Patient-healthcare worker interactions were studied as follows: culture data from each healthcare worker were matched with culture data from the individual patients they cared for on the day of the interaction, the prior sampling date, and the subsequent sampling date.

Definitions of Colonization. Colonization was defined as one or more cultures positive for yeasts from any site. For patients followed for at least three culture episodes, the following definitions were applied: persistent colonization was defined as a culture from at least one site being positive for yeast on every occasion that cultures were performed; probably persistent colonization was defined as a ratio of positive to total cultures performed of between 0.8 and 1 ; intermittent colonization was recorded when patients had more than one culture positive for yeast but were not persistently or probably persistently colonized; transient colonization was defined as only one culture positive for yeast.

Microbiologic Methods. Samples were obtained from the oropharynx and the rectum by use of sterile rayon applicator sticks, which were immediately streaked onto Sabouraud dextrose agar (Difco, USA) containing $50 \mu \mathrm{g} / \mathrm{ml}$ gentamicin sulfate (Sigma, USA) and $50 \mu \mathrm{g} / \mathrm{ml}$ vancomycin (Sigma).

Healthcare workers' hands were sampled using a modified brothbag technique in which both hands were sequentially immersed in $50 \mathrm{ml}$ sterile $0.9 \%$ saline in a sterile zip-lock bag and kneaded for $30 \mathrm{~s}$. The hands were removed from the bag, and the saline remaining in the bag was passed through a $0.45 \mu \mathrm{m}$ filter (Millipore, USA). The filter was carefully placed into $0.5 \mathrm{ml}$ sterile Sabouraud broth and vortexed vigorously. A $0.1 \mathrm{ml}$ aliquot of this fluid was spread with a sterile glass rod onto Sabouraud agar containing $50 \mu \mathrm{g} / \mathrm{ml}$ gentamicin sulfate and $50 \mu \mathrm{g} / \mathrm{ml}$ vancomycin. All plates were incubated at $35^{\circ} \mathrm{C}$ for $72 \mathrm{~h}$, and yeast-like colonies were isolated.

Germ-tube formation for identification of Candida albicans was assessed by incubating a small inoculum of organisms in $0.5 \mathrm{ml}$ of fetal bovine serum at $35^{\circ} \mathrm{C}$ for $2.5 \mathrm{~h}$ and then viewing the preparation under direct microscopy. Yeasts that were germ-tube negative were identified to species level by the API 20C System (Sherwood Medical Products, USA). All isolates were stored at $-70^{\circ} \mathrm{C}$ in Sabouraud broth and glycerol for further studies.

Typing of Yeast Strains. For CHEF typing, DNA was prepared in agarose plugs after yeast cells had been treated with zymolase and lysis with sarcosyl/pronase as described previously [18, 19]. For RAPD polymerase chain reaction (PCR) typing, DNA was extracted from zymolase-treated yeast cells, purified, and resuspended in Tris-EDTA buffer, as described previously [20].

CHEF electrophoresis was performed with agarose plugs containing whole yeast DNA after loading onto a horizontal electrophoresis gel. Chromosomal DNA from Saccharomyces cerevisiae (New England BioLabs, USA) was used as an internal standard. Chromosomal separation was achieved on CHEF DRII (Bio-Rad, USA) equipment for Candida albicans and DR-III equipment for Candida glabrata and Candida parapsilosis using switch-time and voltage parameters appropriate for each species $[18,19,21]$. Gels were stained for $1 \mathrm{~h}$ in $1 \mu \mathrm{g} / \mathrm{ml}$ ethidium bromide and photographed with UV transillumination.

Polymerase Chain Reaction Assay. PCRs were performed in a Robocycler 40 Thermocycler (Stratagene, USA) using a $10 \mathrm{bp}$ primer (CX-5) [20]. The sequence of CX-5 is $5^{\prime}$-ACACTGCTTC$3^{\prime}$. Briefly, the program utilized was as follows: 5 cycles of $94^{\circ} \mathrm{C} \times 30 \mathrm{~s}, 25^{\circ} \mathrm{C} \times 2 \mathrm{~min}, 72^{\circ} \mathrm{C} \times 2 \mathrm{~min} ; 45$ cycles of $94^{\circ} \mathrm{C} \times 30 \mathrm{~s}$, $31^{\circ} \mathrm{C} \times 2 \mathrm{~min}, 72^{\circ} \mathrm{C} \times 2 \mathrm{~min}$, and $1 \mathrm{cycle}$ of $72^{\circ} \mathrm{C} \times 10 \mathrm{~min}$. Typical reactions in $25 \mu \mathrm{l}$, overlaid with mineral oil, were in $25 \mathrm{mM}$ Tris$\mathrm{HCl}, \mathrm{pH} 8.4,50 \mathrm{mM} \mathrm{KCl}, 1.5 \mathrm{mM} \mathrm{MgCl}, 100 \mu \mathrm{l}$ dNTPs, 50 pmoles primer, and $2.5 \mathrm{U}$ Taq DNA polymerase (Bethesda Research Laboratories, USA). Template DNAs were genomic DNA $(<100 \mathrm{ng})$. PCR reaction products were analyzed by ethidium bromide agarose gel electrophoresis ( $2 \%$ agarose). Positive and negative controls were run simultaneously. All PCR studies were carried out in duplicate to ensure stability and reproducibility of the results.

Strain differentiation by CHEF was performed by visual delineation of the banding pattern existing between 225 and $1900 \mathrm{~kb}$. For each isolate, the number of bands and their position relative to the Saccharomyces cerevisiae standard bands were recorded. A strain was considered unique if it had a different number of bands and/or a different position of the bands relative to other previously described banding patterns in our library of CHEF patterns [18, 19]. For PCR assays, visual elucidation of the patterns were recorded; polaroids of gels and autoradiograms were scanned with a Logitech Scanner for printing at $300 \mathrm{dpi}$. On occasion, exposures of lanes that were over- or underloaded were enhanced by the scanning software so that originally visible fragments were not lost in reproduction. 
Statistical Analysis. Data are expressed as mean \pm standard deviation. Data for risk factor analysis were analyzed using Statistical Analysis System 6.12 (SAS Institute, USA). Categorical variables were analyzed with the chi-square or Fisher's exact test; continuous variables were analyzed with the Student's $t$ test. Multivariate analysis was done by stepwise logistic regression in which the limit for entering and removing variables was 0.1 .

\section{Results}

Colonization of Patients with Yeasts. A total of 194 patients (191 men and 3 women) were included in the study; 48 patients were admitted to the medical ICU, 52 to the surgical ICU, and 94 to the thoracic ICU. Patients were followed for a mean of $9 \pm 11$ days (range 4-103 days); the mean length of ICU stay was $11 \pm 12$ days $(9.5 \pm 7$ days in the thoracic ICU; $11 \pm 7.7$ days in the medical ICU; and $13.9 \pm 21$ days in the surgical ICU). The mean age of the study population was $64.1 \pm 10.5$ years (range $27-83$ years).

Patients were sampled for yeast colonization a mean of $3.3 \pm 3.1$ times (range 2-30). Overall, 142 (73\%) patients were positive for yeast on at least one occasion, including $133(69 \%)$ patients with yeast isolated from the oropharynx and $91(47 \%)$ patients with yeast isolated from the rectum. In the medical ICU, 38 (79\%) patients were colonized with yeasts; in the surgical ICU, $38(73 \%)$ patients were colonized; and in the thoracic ICU, 66 (70\%) patients were colonized.

Of the 632 occasions on which samples were taken, yeasts were recovered from either the oropharynx or the rectum on $430(68 \%)$. On $343(80 \%)$ of these occasions, only one species was present, while on $77(18 \%)$ and $10(2 \%)$ occasions, two or more than three species were isolated, respectively. The most common colon-

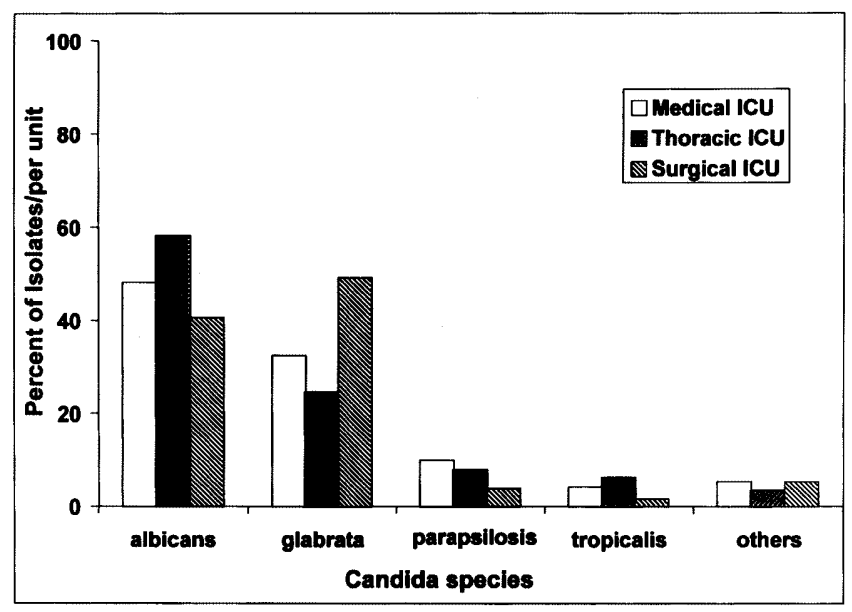

Figure 1 Candida species isolated from patients on 632 occasions according to type of intensive care unit (ICU)

izing species isolated from both the oropharynx and the rectum of patients was Candida albicans (Table 1). Candida glabrata was found as frequently as Candida albicans in the rectum but was less common in the oropharynx. When separated by individual ICUs, Candida albicans accounted for $48.1 \%$ and $58.1 \%$ of all isolates in the medical and thoracic ICUs, respectively (Figure 1). However, in the surgical ICU, only $40.5 \%$ of isolates were Candida albicans; Candida glabrata was more common (49.2\% of isolates). No other major differences were noted in the types of colonizing yeast species among the three units.

Acquisition and Persistence of Yeast Colonization in Patients. There were 92 patients on whom cultures were performed on more than three occasions. Of these 92 patients, $76(82.6 \%)$ were colonized with yeasts. In

Table 1 Yeast species isolated from patients ( $n=632$ occasions) and healthcare workers ( $n=524$ occasions)

\begin{tabular}{|c|c|c|c|c|}
\hline \multirow[t]{2}{*}{ Yeast sp. isolated } & \multicolumn{4}{|c|}{ No. (\%) of positive samples } \\
\hline & Rectum & Oropharynx & Hands & Oropharynx \\
\hline \multicolumn{5}{|l|}{ Patients } \\
\hline C. albicans & $94(14.8)$ & $230(36.4)$ & & \\
\hline C. glabrata & $102(16.1)$ & $126(19.9)$ & & \\
\hline C. parapsilosis & $34(5.4)$ & $11(1.7)$ & & \\
\hline C. tropicalis & $7(1.1)$ & $18(2.8)$ & & \\
\hline C. kefyr & $1(0.2)$ & $9(1.4)$ & & \\
\hline Other $^{\mathrm{a}}$ & $7(1.1)$ & $10(1.6)$ & & \\
\hline No yeasts isolated & $410(64.9)$ & $255(40.3)$ & & \\
\hline \multicolumn{5}{|l|}{ Healthcare workers } \\
\hline C. albicans & & & $25(4.8)$ & $102(19.4)$ \\
\hline C. glabrata & & & $20(3.8)$ & $10(1.9)$ \\
\hline C. parapsilosis & & & $44(8.4)$ & $1(0.2)$ \\
\hline C. tropicalis & & & $1(0.2)$ & 0 \\
\hline C. kefyr & & & 0 & $2(0.4)$ \\
\hline Other $^{\mathrm{a}}$ & & & $13(2.5)$ & 0 \\
\hline No yeasts isolated & & & $440(84.0)$ & $413(78.8)$ \\
\hline
\end{tabular}

${ }^{\mathrm{a}}$ C. lusitaniae, Saccharomyces cerevisiae, C. lipolytica, Trichosporon beigelii, Hansenula and Rhodotorula sp. 
Table 2 Acquisition and persistence of yeast colonization in patients and healthcare workers who had cultures performed on three or more occasions

\begin{tabular}{lcc}
\hline Colonization status & \multicolumn{2}{l}{ No. $(\%)$ of persons } \\
\cline { 2 - 3 } & $\begin{array}{l}\text { Patients } \\
(n=92)\end{array}$ & $\begin{array}{l}\text { Healthcare } \\
\text { workers } \\
(n=58)\end{array}$ \\
& & $6(10.3)$ \\
\hline Persistent colonization & $47(51.1)$ & $6(5.2)$ \\
Probably persistent colonization & $4(4.3)$ & $23(39.7)$ \\
Intermittent colonization & $21(22.8)$ & $7(12.1)$ \\
Transient colonization & $4(4.3)$ & $39(67.3)$ \\
Total colonized & $76(82.5)$ & \\
\hline
\end{tabular}

$60(65.2 \%)$ of them, yeasts were isolated at entry into the study; in $16(17.4 \%)$, yeasts were acquired subsequent to their admission to the ICU; and in another 16 $(17.4 \%)$, yeast colonization did not occur at any time during the study.

Yeasts were persistently and probably persistently isolated from $51(55.4 \%)$ of the 92 total patients, while $21(22.8 \%)$ and $4(4.3 \%)$ patients had only intermittent or transient yeast colonization, respectively (Table 2). On 90 occasions, these 51 patients were simultaneously colonized with the same species of yeast in the rectum and the oropharynx. Strain delineation of 39 randomly selected pairs of Candida albicans and Candida glabrata recovered on these 90 occasions revealed that the strains from the rectum and the oropharynx were similar in $27(69.2 \%)$ of 39 pairs tested.

Candida albicans was most often associated with persistent or probably persistent colonization. Fifty-three percent of patients colonized with Candida albicans had persistent or probably persistent colonization, whereas only $25.5 \%$ and $11.1 \%$ of patients colonized with Candida glabrata or Candida parapsilosis, respec- tively, had persistent or probably persistent colonization. Strain delineation was performed on isolates recovered from 40 patients who had persistent or probably persistent colonization with Candida albicans (29 patients) or Candida glabrata (11 patients). Twenty-two (75.9\%) of the 29 patients colonized with Candida albicans and $8(73.7 \%)$ of 11 patients colonized with Candida glabrata were found to be persistently colonized with the same strain throughout their ICU stay.

The mean time until colonization was $6 \pm 1.6$ days for the 16 patients who became colonized after admission to the ICU. The organism first acquired was Candida glabrata in six patients, Candida albicans in five, Candida parapsilosis in two, and mixtures of Candida glabrata with other yeasts in three patients. Once acquired, Candida glabrata persisted in five of nine patients, while Candida albicans persisted in only two of eight patients.

Risk Factors for Colonization of Patients with Yeasts. Evaluation of risk factors present on admission to the ICU revealed that only bacterial infections within the previous month were associated with yeast colonization at either site $(P=0.002)$ (Table 3$)$. Renal failure was more common in colonized patients, but differences were not statistically different. Multivariate analysis confirmed that the only factor significantly associated with fungal colonization was prior bacterial infection (relative risk $=4.2[1.6-10.6], P=0.002$ ).

Fungemia Episodes. One patient developed fungemia on two occasions during the 6-month study period. This patient, whose length of stay in the surgical ICU was 103 days, had Candida glabrata fungemia followed 3 months later by Candida albicans fungemia. He had probably persistent colonization with Candida glabrata in both the oropharynx and the rectum throughout the study period, but Candida albicans was never isolated from either site prior to the fungemic episodes.

Table 3 Factors associated with colonization of 194 ICU patients by yeasts

\begin{tabular}{|c|c|c|c|c|}
\hline \multirow[t]{2}{*}{ Characteristic } & \multicolumn{2}{|c|}{ No. (\%) of patients } & \multirow{2}{*}{$\begin{array}{l}\text { Relative risk } \\
(95 \% \text { CI })\end{array}$} & \multirow[t]{2}{*}{$P$ value } \\
\hline & $\begin{array}{l}\text { Colonized } \\
(n=142)\end{array}$ & $\begin{array}{l}\text { Not colonized } \\
(n=52)\end{array}$ & & \\
\hline Age $($ mean $\pm S D)$ & $64.5 \pm 10.9$ & $63.4 \pm 9.3$ & & 0.54 \\
\hline Prior intravenous antibiotics ${ }^{a}$ & $136(95.8)$ & $47(90.4)$ & $1.8(0.8-3.9)$ & 0.15 \\
\hline Prior surgical procedure ${ }^{\mathrm{b}}$ & $105(73.9)$ & $44(84.6)$ & $0.6(0.3-1.1)$ & 0.12 \\
\hline Diabetes mellitus & $53(37.3)$ & $15(28.8)$ & $1.3(0.8-2.2)$ & 0.27 \\
\hline Cancer & $30(21.1)$ & $14(26.9)$ & $0.8(0.4-1.3)$ & 0.39 \\
\hline Alcoholism & $24(16.9)$ & $9(17.3)$ & $1.0(0.5-1.8)$ & 0.95 \\
\hline Neutropenia & $1(0.7)$ & 0 & $1.1(0.1-12.7)$ & 0.55 \\
\hline Renal failure & $9(6.3)$ & 0 & $5.6(0.4-84.9)$ & 0.06 \\
\hline Corticosteroid therapy ${ }^{\mathrm{b}}$ & $8(5.6)$ & $2(3.8)$ & $1.4(0.4-4.5)$ & 0.62 \\
\hline Parenteral nutrition $^{\mathrm{a}}$ & $13(9.2)$ & $2(3.8)$ & $2.1(0.6-6.8)$ & 0.22 \\
\hline Bacterial infection $^{\mathrm{a}}$ & $37(26.1)$ & $3(5.8)$ & $4.2(1.7-10.6)$ & 0.002 \\
\hline
\end{tabular}

\footnotetext{
${ }^{a}$ Within preceding month

${ }^{\mathrm{b}}$ Within preceding 3 months
} 
Colonization of Healthcare Workers with Yeasts. A total of 63 healthcare workers (54 women and 9 men) were followed for a mean of $132 \pm 52$ days and were sampled for yeast colonization on $8.3 \pm 4.5$ occasions (range 1-22). Among them, 42 (66.7\%) were positive for yeast on at least one occasion; $27(42.9 \%)$ had oropharyngeal colonization and $30(47.6 \%)$ had hand colonization.

Yeasts were isolated from either the oropharynx or the hands of healthcare workers on $166(31.7 \%)$ of the 524 total occasions that samples were taken. On 135 $(81.3 \%)$ of these occasions, only one species was present, whereas on $26(15.7 \%)$ and $5(3 \%)$ occasions, two or more than three species were isolated, respectively. Overall, $16 \%$ of hand samples and $21 \%$ of oropharyngeal samples yielded yeasts. Candida albicans was the species most commonly isolated from the oropharynx of healthcare workers $(19.4 \%)$, whereas Candida parapsilosis was the species most frequently isolated from hands (8.4\%) (Table 1). Candida glabrata was infrequently isolated from either site in healthcare workers.

Of the $58(92.1 \%)$ healthcare workers who had samples taken on more than three occasions, $6(10.3 \%)$ were persistently colonized with yeasts and $3(5.2 \%)$ were probably persistently colonized; $23(39.7 \%)$ and 7 $(12.1 \%)$ had intermittent and transient colonization, respectively (Table 2). Persistent colonization was documented in the oropharynx only, not on the hands, of healthcare workers. Persistent or probably persistent colonization by one yeast species occurred in only three healthcare workers, while six had more than one Candida species isolated over time. Only two healthcare workers were persistently colonized by the same species simultaneously in the oropharynx and on the hands.

Although there were no healthcare workers whose hands were persistently colonized by yeast, three had probably persistent colonization and two others had multiple positive cultures from hands, but by strict definition they were judged to be intermittent carriers. Four of these five healthcare workers carried multiple different yeast species on their hands over time and three had multiple species isolated on most sampling occasions. Strain delineation showed that one strain persisted over time in two of four healthcare workers colonized with Candida albicans, one of three healthcare workers colonized with Candida glabrata, and one of three healthcare workers colonized with Candida parapsilosis. The one healthcare worker who had Candida parapsilosis only isolated on each sampling occasion had four different strain types isolated. There were 11 healthcare workers in whom Candida albicans was frequently isolated from the oropharynx over time; in contrast to the results noted with hand carriage, 9 of these 11 healthcare workers had the same strain of
Candida albicans isolated from the oropharynx over time.

Transfer of Yeasts Between Healthcare Workers and Patients. In order to investigate the possibility of yeasts being transferred between healthcare workers and patients, we studied each interaction $(n=123)$ between a healthcare worker and a patient in which the healthcare worker had yeasts isolated and the patient had cultures obtained at, right before, or right after the time of the interaction. For 56 of these 123 patienthealthcare worker interactions, the same species of yeast was isolated (35 Candida albicans, 12 Candida parapsilosis, and 9 Candida glabrata). For those 21 interactions in which Candida glabrata or Candida parapsilosis were found in both healthcare workers and patients, the strains obtained from healthcare workers were not similar to those obtained from patients.

CHEF typing showed that for 13 of the 35 interactions yielding Candida albicans, the same strain was isolated from both the healthcare worker and the patient. RAPD analysis differentiated 8 of these 13 as different strains, with 5 strains being similar by both techniques (Figure 2). Thus, for $5(4.1 \%)$ of 123 interactions, evidence for possible transmission was obtained. None of these interactions was between a healthcare worker and any of the 16 patients who entered the ICU without prior Candida colonization. Thus, no direct evidence was obtained to link initial acquisition of yeasts upon entry into the ICU with transmission from a healthcare worker. In fact, for these five interactions, the patient was found to carry the strain before the healthcare worker in three instances, and in two other instances, both the healthcare worker and the patient were found to carry the same strain for the first time on the same day.

\section{Discussion}

Over the past decade nosocomial infection with Candida species has emerged as a major problem for patients in acute-care hospitals. The recent trend of an increase in the incidence of nosocomial candidemia is especially worrisome, with a fivefold increase having been noted among hospitals participating in the National Nosocomial Infections Surveillance system in the USA [1], a 2.3-fold increase recorded in the Netherlands [22], and a 27-fold increase noted in Taiwan [23]. The greatest risk for fungemia appears to be in the ICU with certain units (e.g., burn/trauma and neonatal units) showing the highest rates [1, 24].

In the present study, colonization of ICU patients with Candida spp. was common, occurring at least once in $73 \%$ of patients, a figure not dissimilar to that found in prior studies $[5,14,25]$. Most patients who entered the ICU were already colonized by their own Candida 
Figure 2 RAPD fingerprinting of Candida albicans isolates recovered from healthcare worker-patient interactions. Lanes 1 and 2 represent isolates from a healthcare worker and a patient who did not share a similar strain. Lanes 3 and 4 are isolates from a healthcare workerpatient interaction in which the strains appeared similar. Lanes 5 through 8 contain isolates from 2 healthcare workers (lanes 5 and 8 ), each of whom shared a similar strain with a patient for whom they cared (lanes 6 and 7, respectively). Lanes 9 and 10 are patient isolates recovered during interactions with the healthcare worker represented in lane 11; no similarities are noted. The lane on the far right contains the size standards (in $\mathrm{Kb}$ ) that were generated by EcoR1-HindIII digestion of bacteriophage lambda

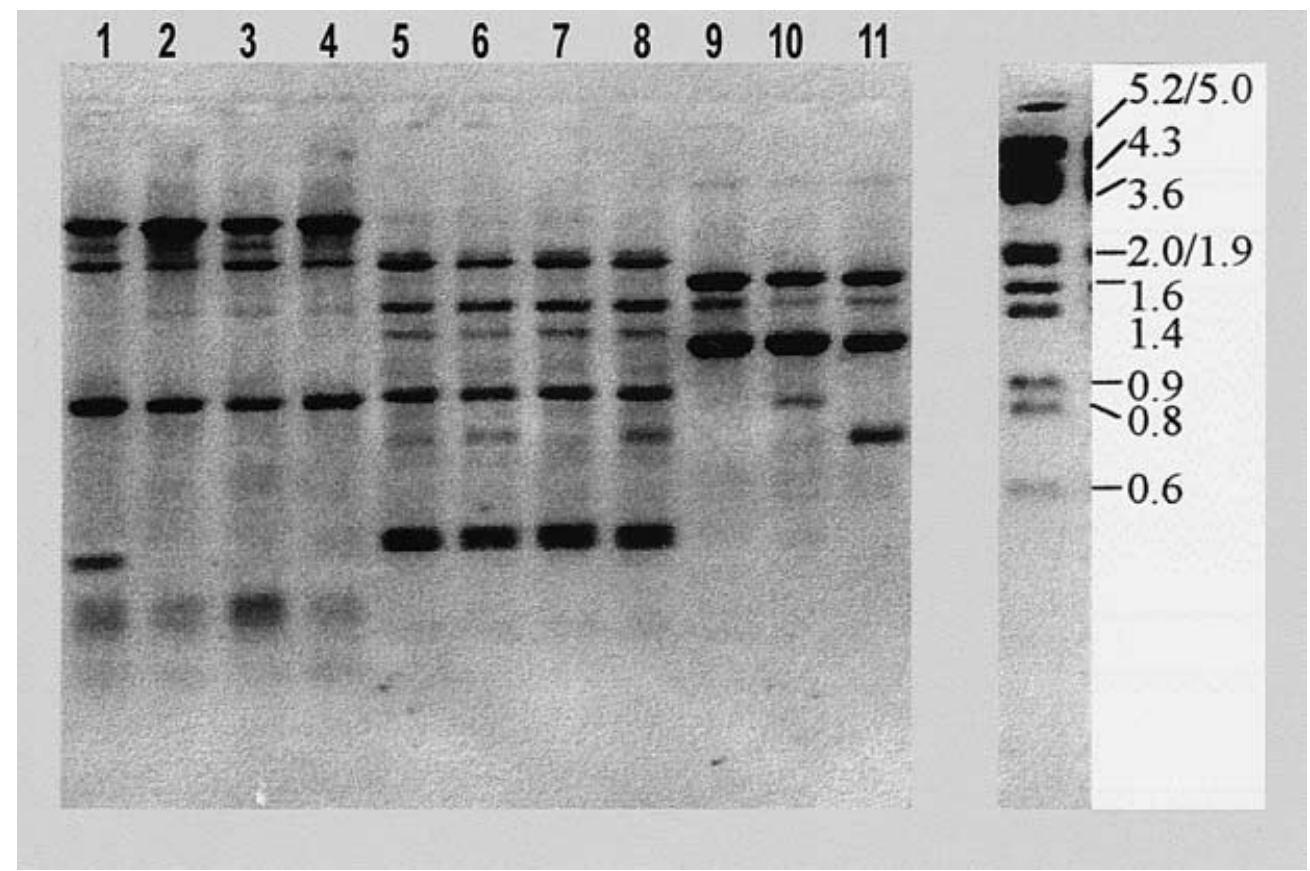

strain, mostly Candida albicans. Colonization with this same strain persisted in the majority of patients, verifying results of a prior study [5].

Most factors typically associated with an increased risk of developing candidemia $[1,26,27]$ did not appear to contribute to Candida colonization. Multivariate analysis revealed that bacterial infection within the previous month was the only factor associated with an increased risk of yeast colonization. We did not find, as others have, an association between prior antibiotic therapy and yeast colonization [15, 28]. However, the extensive use of antibiotics in this population, often without a documented bacterial infection, could be responsible for this lack of relationship to colonization.

Current understanding of the epidemiology of acquisition and infection with Candida species in the ICU setting is incomplete. Several investigators have studied the unique situation afforded by the neonatal ICU, in which initial acquisition of yeasts by a Candida-naive patient can be assessed [29-31]. Reef et al. [30] found that all neonates were free of yeast colonization at the time of birth, while others have shown that colonization was present at first sampling in a small percentage of neonates [31]. Those neonates who did become colonized with Candida spp., especially Candida parapsilosis, appeared to acquire yeasts most often from the ICU setting and less often from their mothers [29-31]. However, none of these studies on neonates assessed the carriage of yeasts by healthcare workers and the role which carriage might play in transmission to neonates.
In adults, most of whom enter an ICU already colonized with yeasts, the epidemiology of Candida acquisition differs from that found for neonates. Presumably, exogenous strains have to compete with those endogenous Candida strains already present. One source of exogenous yeast acquisition could be the hands of healthcare workers. Although $48 \%$ of healthcare workers in this study had yeasts on their hands, none had persistent carriage and only five had frequent carriage at this site. Other investigators have found the rate of carriage of Candida on healthcare workers' hands to vary from 0 to $58 \%[13,15,16,25,28,32-34]$. Of the five healthcare workers whose hands were frequently colonized with Candida in our study, only one was colonized with a single species (Candida parapsilosis) over time, and this person had different strains of that species on each occasion. All of the healthcare workers with frequent colonization had either multiple species isolated at each sampling or had multiple strains of the same species isolated over time. Thus, it appears that hand carriage of Candida by healthcare workers is usually short-lived. One recent study that assessed persistence of carriage during an ICU shift found that nurses initially had Candida parapsilosis on their hands and acquired Candida albicans only after patient care activities [33].

The most common species isolated from the hands of healthcare workers was Candida parapsilosis, a finding consistent with that noted by several investigators [13, 16] but different from that noted by others [25]. In spite of the common presence of Candida parapsilosis on healthcare workers' hands and data showing that persistence on hands may be longer for Candida parap- 
silosis than other species [35], Candida parapsilosis was uncommonly noted in our patients, rarely persisted when it did occur, and no link between healthcare worker carriage and patient acquisition could be found.

Although transmission of Candida parapsilosis in nonoutbreak settings has been postulated [36], proof of transmission has been lacking. Future studies assessing transmission of Candida parapsilosis might be most fruitfully performed in two settings: (i) neonatal ICUs, in which the rates of colonization and serious infection with Candida parapsilosis appear to be much higher than those noted in adult ICUs [37-39]; and (ii) outbreak settings, in which contamination of central intravenous catheters or other devices may be related to the carriage of yeasts on the hands of healthcare workers $[6,29]$.

In our study, Candida glabrata was found to be most common in the surgical ICU. This finding is similar to that noted in a large multicenter study of colonization and infection in ICUs, in which Candida glabrata was more common among patients in a surgical ICU than among those in neonatal or cardiothoracic ICUs [39]. Among those patients who were not colonized with yeasts on admission to the ICU, acquisition of Candida glabrata was more common than acquisition of Candida albicans. However, healthcare workers were infrequently colonized with Candida glabrata on their hands, and no evidence of transmission of Candida glabrata from a healthcare worker to a patient was found. Thus, possible mechanisms of acquisition of Candida glabrata have not been specifically elucidated by this study. Prior studies in a medical ICU noted environmental contamination by Candida glabrata [28]. It is possible that more frequent sampling of healthcare workers' hands in our study could have revealed transient contamination of hands with this species. It should be noted that fluconazole, restricted to use by the Infectious Disease Section of our hospital, was dispensed infrequently in the ICUs.

In the absence of an outbreak, the possibility of yeasts being transmitted between healthcare workers and patients remains controversial. Hunter et al. [25], using insensitive strain typing techniques, found little to support cross-transmission. Using a more sensitive restriction enzyme analysis method, Vazquez et al. [15, 28] found possible cross-transmission of both Candida albicans and Candida glabrata in a medical ICU and a bone marrow transplant unit. However, a link between colonization of healthcare workers' hands and transmission was not made in those studies, possibly because sensitive hand sampling techniques were not performed over time.

Our study, which utilized multiple samplings over time, was conducted in a non-outbreak setting to assess whether usual healthcare worker-patient interactions might play a role in the transmission of yeasts. On only $5(4.1 \%)$ of 123 occasions were both the healthcare worker and the patient for whom care was provided found to carry the same strain of yeast. This finding, obtained using two different typing methods [40], supports prior studies showing possible cross-transmission of yeast strains from healthcare workers to patients $[11,15,28,36]$, but it does not prove that crosstransmission occurred. Further studies focusing on daily healthcare worker-patient interactions, taking into account such factors as hand washing, glove use, and use of invasive devices, should help define further the role that healthcare worker-patient interactions play in the transmission of yeasts.

Acknowledgements The authors acknowledge the expert technical assistance of M. Ramsey and L. Zarins. This study was supported by a grant from Pfizer-Roerig.

\section{References}

1. Jarvis WR: Epidemiology of nosocomial fungal infections, with emphasis on Candida species. Clinical Infectious Diseases (1995) 20:1526-1530

2. Pfaller MA, Jones RN, Messer SA, Edmond MB, Wenzel RP and the SCOPE Participant Group: National surveillance of nosocomial blood stream infection due to species of Candida other than Candida albicans: Frequency of occurrence and antifungal susceptibility in the SCOPE program. Diagnostic Microbiology and Infectious Diseases (1998) 30:121-129

3. Voss A, Hollis RJ, Pfaller MA, Wenzel RP, Doebbeling BN: Investigation of the sequence of colonization and candidemia in nonneutropenic patients. Journal of Clinical Microbiology (1994) 32:975-980

4. Reagan DR, Pfaller MA, Hollis RJ, Wenzel RP: Characterization of the sequence of colonization and nosocomial candidemia using DNA fingerprinting and a DNA probe. Journal of Clinical Microbiology (1990) 28:2733-2738

5. Pittet D, Monod M, Filthuth I, Frenk E, Suter PM, Auckenthaler R: Contour-clamped homogeneous electric field gel electrophoresis as a powerful epidemiologic tool in yeast infections. American Journal of Medicine (1991) 91, Supplement 3:256-263

6. Weems JJ, Chamberland ME, Ward J, Willy M, Padhye AA, Solomon SL: Candida parapsilosis fungemia associated with parenteral nutrition and contaminated blood pressure transducers. Journal of Clinical Microbiology (1987) 25:1029-1032

7. Isenberg HD, Tucci V, Cintron F, Singer C, Weinstein GS, Tyras DH: Single-source outbreak of Candida tropicalis complicating coronary bypass surgery. Journal of Clinical Microbiology (1989) 27:2426-2428

8. Pertowski CA, Baron RC, Lasker BA, Werner SB, Jarvis WR: Nosocomial outbreak of Candida albicans sternal wound infections following cardiac surgery traced to a scrub nurse. Journal of Infectious Diseases (1995) 172:817-822

9. Burnie JP, Odds FC, Lee W, Webster C, Williams JD: Outbreak of systemic Candida albicans in an intensive care unit caused by cross infection. British Medical Journal (1985) 290:746-748

10. Betremieux P, Chevrier S, Quindos G, Sullivan D, Polonelli L, Guiguen C: Use of DNA fingerprinting and biotyping methods to study a Candida albicans outbreak in a neonatal intensive care unit. Pediatric Infectious Disease Journal (1994) 13:899-905 
11. Fowler SL, Rhoton B, Springer SC, Messer SA, Hollis RJ, Pfaller MA: Evidence for person-to-person transmission of Candida lusitaniae in a neonatal intensive-care unit. Infection Control and Hospital Epidemiology (1998) 19:343-345

12. Vaudry WL, Tierney AJ, Wenman WM: Investigation of a cluster of Candida albicans infections in a neonatal intensive care unit. Journal of Infectious Diseases (1988) 158:1376-1379

13. Diekema DJ, Messer SA, Hollis RJ, Wenzel RP, Pfaller MA: An outbreak of Candida parapsilosis prosthetic valve endocarditis. Diagnostic Microbiology and Infectious Diseases (1997) 29:147-153

14. Tran LT, Auger P, Marchand R, Carrier M, Pelletier C: Epidemiological study of Candida spp. colonization in cardiovascular surgical patients. Mycoses (1997) 40:169-173

15. Vazquez JA, Sanchez V, Dmuchowski C, Dembry LM, Sobel JD, Zervos MJ: Nosocomial acquisition of Candida albicans: An epidemiologic study. Journal of Infectious Diseases (1993) 168:195-201

16. Strausbaugh LJ, Sewell DL, Ward TT, Pfaller MA, Heitzman T, Tjoelker R: High frequency of yeast carriage on hands of hospital personnel. Journal of Clinical Microbiology (1994) 32:2299-2300

17. Espinel-Ingoff A, Vazquez JA, Boikov D, Pfaller MA: Evaluation of DNA-based typing procedures for strain categorization of Candida sp. Diagnostic Microbiology and Infectious Diseases (1998) 33:231-239

18. Sangeorzan JA, Zervos MJ, Donabedian S, Kauffman CA: Validity of contour-clamped homogeneous electric field electrophoresis as a typing system for Candida albicans. Mycoses (1995) 38:29-36

19. Sangeorzan JA, Bradley SF, He X, Zarins LT, Ridenour GL, Tiballi RN, Kauffman CA: Epidemiology of oral candidiasis in HIV-infected patients: colonization, infection, treatment, and emergence of fluconazole resistance. American Journal of Medicine (1994) 97:339-346

20. Steffan P, Vazquez JA, Boikov D, Xu C, Sobel JD, Akins RA: Identification of Candida species by randomly amplified polymorphic DNA fingerprinting of colony lysates. Journal of Clinical Microbiology (1997) 35:2031-2039

21. Khattak MN, Burnie JP, Matthews RC, Oppenheim BA: Clamped homogeneous electric field gel electrophoresis typing of Torulopsis glabrata isolates causing nosocomial infections. Journal of Clinical Microbiology (1992) 30:2211-2215

22. Voss A, Kluytmans JAJW, Koeleman JGM, Spanjaard L, Vandenbroucke-Grauls CMJE, Verbrugh HA, Vos MC, Weersink AYL, Hoogkamp-Korstanje JA, Meis JFGM: Occurrence of yeast bloodstream infections between 1987 and 1995 in five Dutch university hospitals. European Journal of Clinical Microbiology \& Infectious Diseases (1996) 15:909-912

23. Chen Y-C, Chang S-C, Sun C-C, Yan L-S, Hsieh W-C, Luh $\mathrm{K}-\mathrm{T}$ : Secular trends in the epidemiology of nosocomial fungal infections at a teaching hospital in Taiwan, 1981 to 1993. Infection Control and Hospital Epidemiology (1997) 18:369-375

24. Fridkin SK, Jarvis WR: Epidemiology of nosocomial fungal infections. Clinical Microbiology Reviews (1996) 9:499-511

25. Hunter PR, Harrison GAJ, Fraser CAM: Cross-infection and diversity of Candida albicans strain carriage in patients and nursing staff on an intensive care unit. Journal of Medical and Veterinary Mycology (1990) 28:317-325

26. Wey SB, Mori M, Pfaller MA, Woolson FR, Wenzel RP: Risk factors for hospital-acquired candidemia. Archives of Internal Medicine (1989) 149:2349-2353
27. Bross J, Talbot GH, Maislin G, Horwitz S, Stram BL: Risk factors for nosocomial candidemia: a case-control study. American Journal of Medicine (1989) 87:614-620

28. Vazquez JA, Dembry LM, Sanchez V, Vazquez MA, Sobel JD, Dmuchowski C, Zervos MJ: Nosocomial Candida glabrata colonization: an epidemiologic study. Journal of Clinical Microbiology (1998) 36:421-426

29. Ruiz-Diez B, Martinez V, Alvarez M, Rodriguez-Tudela JL, Martinez-Suarez JV: Molecular tracking of Candida albicans in a neonatal intensive care unit: long-term colonizations versus catheter-related infections. Journal of Clinical Microbiology (1997) 35:3032-3036

30. Reef SE, Lasker BA, Butcher DS, McNeil MM, Pruitt R, Keyserling H, Jarvis WR: Nonperinatal nosocomial transmission of Candida albicans in a neonatal intensive care unit: prospective study. Journal of Clinical Microbiology (1998) 36:1255-1259

31. Waggoner-Fountain LA, Walker MW, Hollis RJ, Pfaller MA, Ferguson JE, Wenzel RP, Donowitz LG: Vertical and horizontal transmission of unique Candida species to premature newborns. Clinical Infectious Diseases (1996) 22:803-808

32. Strausbaugh LJ, Sewell DL, Tjoelker RC, Heitzman T, Webster T, Ward TT, Pfaller MA: Comparison of three methods for recovery of yeasts from hands of health-care workers. Journal of Clinical Microbiology (1996) 34:471-473

33. Verdwyn Lunel FM, Meis JFGM, Voss A: Nosocomial fungal infections: candidemia. Diagnostic Microbiology and Infectious Diseases (1999) 34:213-220

34. Rangel-Frausto MS, Wiblin T, Blumberg HM, Saiman L, Patterson J, Rinaldi M, Pfaller M, Edwards JE, Jarvis W, Dawson J, Wenzel RP, and the NEMIS Study Group: National epidemiology of mycoses survey (NEMIS): variations in rate of bloodstream infections due to Candida species in seven surgical intensive care units and six neonatal intensive care units. Clinical Infectious Diseases (1999) 29:253-258

35. Rangel-Frausto MS, Houston AK, Bale MJ, Fu C, Wenzel RP: An experimental model for study of Candida survival and transmission in human volunteers. European Journal of Clinical Microbiology \& Infectious Diseases (1994) 13:590-595

36. Sanchez V, Vazquez JA, Barth-Jones D, Dembry L, Sobel JD, Zervos MJ: Nosocomial acquisition of Candida parapsilosis: an epidemiological study. American Journal of Medicine (1993) 94:577-582

37. Levy I, Rubin LG, Vasishtha SV, Tucci V, Sood SK: Emergence of Candida parapsilosis as the predominant species causing candidemia in children. Clinical Infectious Diseases (1998) 26:1086-1088

38. Vazquez JA, Boikov D, Boikov SG, Dajani AS: Use of electrophoretic karyotyping in the evaluation of Candida infections in a neonatal intensive care unit. Infection Control and Hospital Epidemiology (1997) 18:32-37

39. Pfaller MA, Messer SA, Houston A, Rangel-Frausto MS, Wiblin T, Blumberg HM, Edwards JE, Jarvis W, Martin MA, Neu HC, Saiman L, Patterson JE, Dibb JC, Roldan CM, Rinaldi MG, Wenzel RP: National Epidemiology of Mycoses Survey: a multicenter study of strain variation and antifungal susceptibility among isolates of Candida species. Diagnostic Microbiology and Infectious Diseases (1998) 31:289-296

40. Voss A, Pfaller MA, Hollis RJ, Melchers WJG, Mei JFGM: Evaluation of the discriminatory power of pulsed-field gel electrophoresis and PCR fingerprinting for epidemiologic typing of Candida species. Clinical Microbiology \& Infection (1998) 4:82-87 